\title{
Analysis of physical properties of the HAT-P-12b planetary system
}

\author{
Sommkorn Chaiwarakorn $^{a}$, Wiraporn Maithong ${ }^{\mathrm{b}}$, Nursiba Musor ${ }^{\mathrm{c}}$ and Suhaimee Samae $^{\mathrm{d}}$ \\ a,c Physics Program, Faculty of Science Technology and Agriculture, Yala Rajabhat University, Yala, Thailand, 95000, \\ *sommkorn.c@yru.ac.th \\ ${ }^{\mathrm{b}}$ Department of Physics and General Science, Faculty of Science and Technology, Chiang Mai Rajabhat University, Chiang \\ Mai, Thailand, 50300wiraporn_mai@cmru.ac.th \\ ${ }^{\mathrm{c}}$ Thammawittaya Mulniti School, Yala, Thailand, 95000
}

Article History: Received: 10 November 2020; Revised 12 January 2021 Accepted: 27 January 2021; Published online: 5 April 2021

\begin{abstract}
The exoplanet HAT-P-12b was observed at the Sierra Remote Observatories by a 0.7 meters diameter reflective robotic telescope CDK700 with the CCD photometric technique. This research using 317 images of the HAT-P-12b on April 14,2017 . They were analyzed the photometric photometry and calculated the physical properties by the AstroImageJ program. The results show that the radius of the exoplanet HAT-P-12b is $0.951 \mathrm{RJ}$, orbital period P about 3.2105 days. The center of page traverse Tc is 2457857.166110000 (BJD) and the total time of traverse T is 0.093519 days. The Star and Exoplanet View program was created the HAT-P-12b model that the decreasing brightness with 1.94864 percent the average distance between the exoplanet HAT-P-12b and the center is 0.0388 AU.
\end{abstract}

Keywords: HAT-P-12b, exoplanet, physical properties

\section{Introduction}

Planet that is outside the solar system (Extrasolar-planet) or shortly known as exoplanets (Exoplanet) was discovered in 1995 by two scientists, Meyer and Queloz. The 51Peg b is a planet with a mass is 0.7 Jupiter-mass. It has a short orbit about 4 days and an average distance is 0.05 AU from its mother star. Then, the discovery of extrasolar planets has increasing. In the present the exoplanets more than 3,400 system were discovered and almost were look like the Jupiter diameter.

The objectives of the study about the exoplanet are explored the planets outside the solar system or orbit planets around other stars and compare the characteristics of the exoplanets and in our solar system. For example, the most planets in our solar system have relatively orbit and there are the large planets such as Jupiter or Saturn are the outer planets. Furthermore, the most important goal of the exploration is the opportunity to discover Earthlike planets that both of size and distance from the main sequence stars of the same spectrum as the Sun. The simple observation of the exoplanet is the light curve analysis. When the exoplanet transits the mother's star, the stellar flux is increasing that as shown in Fig.1.

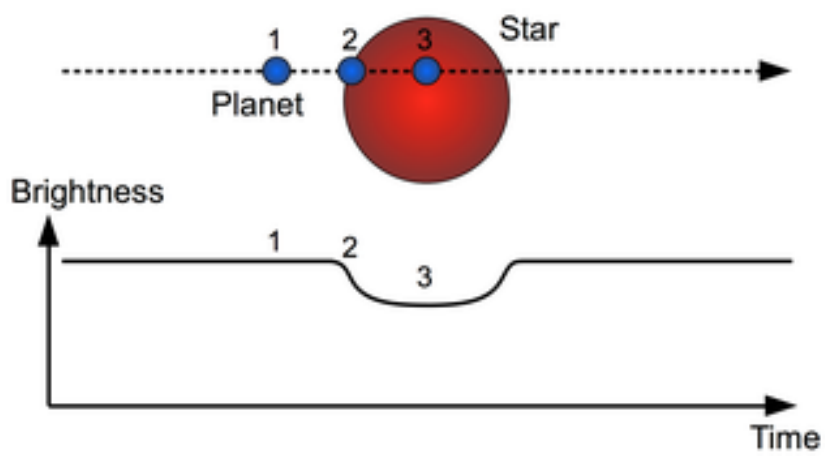

Fig. 1 The light curve of the exoplanet.

(Source; wikipedia.org [1]) 
Furthermore, the spectroscopic study used to determine the approximate mass of the planet that means the estimate its size is accurately and precisely. However, the 78 percent of the exoplanets were discovered and confirmed by the exoplanet transit detection.

In this paper, the exoplanet HAT-P-12b was observed by CCD photometric method analyzed by the transit method. In this work using free software called AstroImageJ (Collins et al, 2015) to measure the photometric and generate its light curve. The AstroImageJ can automatically adjust the model to match the data point and report its physical properties.

\section{Research Objectives}

1.To determine the physical origin properties of the exoplanet HAT-P-12b.

2. To create models in exoplanet experiments.

\section{Experimental}

The equations and parameters of the planetary system were studied from Seager and Mall en-Ornelas optical graph analysis (2003). The duration time of the transposition (T14) which necessary to calculate the basic of the physical parameters that obtained from the observation. The AstroImageJ use to computed the data from the light curve (Fig.5), that will be described as follows.

from

The value $(\mathrm{Rp} / \mathrm{R} *)^{2}$ is the depth of the passage Which can be expressed as a percentage that compute

$$
\Delta F \equiv \frac{F_{\text {notransit }}-F_{\text {transit }}}{F_{\text {notransit }}}=\left(\frac{R_{p}}{R_{*}}\right)^{2}
$$

Where $\mathrm{Rp}$ is the radius of the planet.

$\mathrm{R} *$ is the radius of the star.

The radius of the planet $\mathrm{Rp}$ is show in times of the Jupiter radius that is,

$$
\frac{\mathrm{R}_{\mathrm{P}}}{\mathrm{R}_{\odot}}=\frac{\mathrm{R}_{*}}{\mathrm{R}_{\odot}} \sqrt{\Delta \mathrm{F}}=\left[\mathrm{k}^{1 / \mathrm{x}} \frac{\rho_{*}}{\rho_{\odot}}\right]^{\frac{\times}{(1-3 \times)}} \sqrt{\Delta \mathrm{F}}
$$

So,

$\frac{R_{P}}{R_{\odot}}=\left(\frac{\rho_{*}}{\rho_{\odot}}\right)^{-0.57} \sqrt{\Delta F}$

The a / $\mathrm{R}^{*}$ is the half major axis of the orbit. The term for the stellar radius is given by

$\frac{a}{R_{*}}=\left[\frac{(1+\sqrt{\Delta F})^{2}-b^{2}\left(1-\sin ^{2} \frac{T_{14} \pi}{P}\right)}{\sin ^{2} \frac{T_{14} \pi}{P}}\right]^{1 / 2}$

$\mathrm{T}_{14}$ is the time of the transit.

$\mathrm{P}$ is the orbital period.

$\Delta \mathrm{F}$ is the differential flux when the exoplanet is transit.

Thus, the time of the transit duration, or the duration from the first to the last touch of the shield, when Rp and a are much less than $\mathrm{R}^{*}$ that is,

$$
T_{14}=\frac{R_{*}}{a} \frac{P}{\pi}
$$

In this study, the exoplanet HAT-P-12b is in the HAT-P-12 system in the Canes Venatici constellation. It was observed on April 14, 2017 by a 0.7 meters diameter reflective robotic telescope with the CCD and UBV filters system at the sierra remote observatories, the National Astronomical Research Institute (Public Organization). The position of the observatory is the sierra Nevada mountain range, California, USA. The latitude and longitude are $37.07{ }^{0} \mathrm{~N}$ and $119.4{ }^{0} \mathrm{~W}$, respectively. It is $1,405.128$ meters high from mean sea level. The sample of the HAT-P$12 \mathrm{~b}$ as shown in Fig.2. 


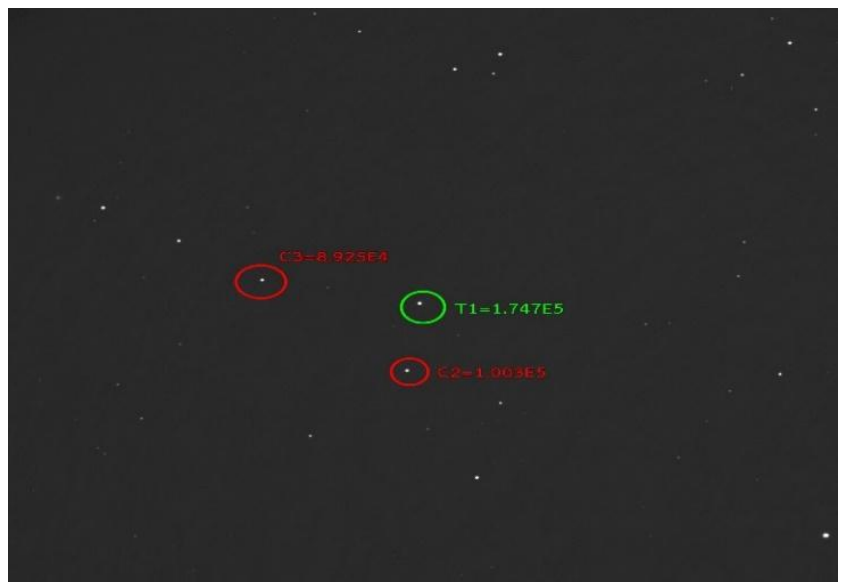

Fig. 2 The image of the planetary system HAT-P-12.

From the Fig. 2 the GSC2 N130301284 and GSC2 N130301294 were used to the references star. The basic information as shown in Table 1.

Table 1. The star information from this observation.

\begin{tabular}{lll}
\hline Object & R.A. & Dec. \\
\hline HAT-P-12 & 135733.47 & +432936.6 \\
GSC2 N130301284 & 135724.99 & +433134.0 \\
GSC2 N130301294 & 135716.64 & +432653.6 \\
\hline
\end{tabular}

The 317 observation images on April 14, 2017 were analyzed and constructed the light curve of the exoplanet HAT-P-12b as shown in Fig.3.

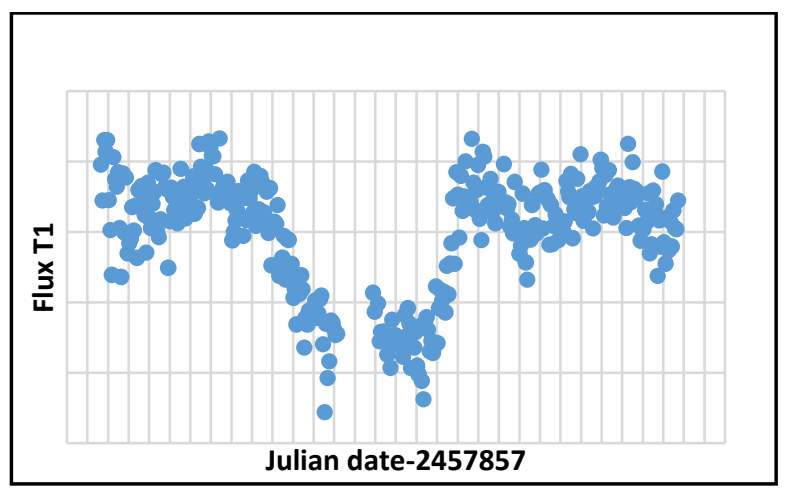

Fig. 3. The light curve of the exoplanet HAT-P-12b

\section{Results And Discussion}

The photometry technique was used to analyzed the exoplanet HAT-P-12b observation images. The syntactic light curve (strength line) was adjusted and corresponding to the data point (dot line) as shown in Fig.4. 


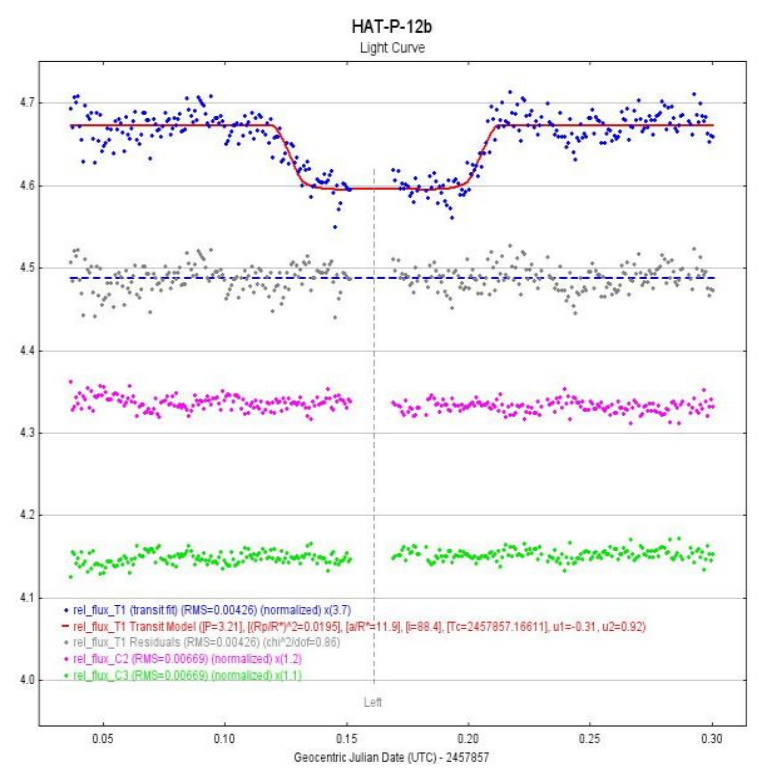

Fig. 4. The syntactic light curve with the exoplanet HAT-P-12b

The physical properties that consistent with the light curve as shown in the Table 2.

Table 2. The best parameter of the exoplanet HAT-P-12b.

\begin{tabular}{ll}
\hline \multicolumn{1}{c}{ Parameter } & Value \\
\hline $\mathrm{M}^{*}\left(\mathrm{M}_{\text {sun }}\right)$ & 0.645 \\
$\mathrm{R}^{*}\left(\mathrm{R}_{\text {sun }}\right)$ & 0.701 \\
$\mathrm{Rp}\left(\mathrm{R}_{\mathrm{Jop}}\right)$ & 0.41 \\
$\left(\mathrm{Rp} / \mathrm{R}_{*}\right)^{\wedge} 2$ & 0.019486400 \\
$\rho^{*}(c g s)$ & 2.603 \\
$\mathrm{a} / \mathrm{R}_{*}$ & 11.939 \\
$\mathrm{~T}_{\mathrm{c}}(\mathrm{BJD})$ & 2457857.166110000 \\
$\mathrm{Inclination}(\mathrm{i})$ & 88.4 \\
$\mathrm{~T} 14$ & 0.093519 \\
Planet Radius & 0.95 \\
& \\
b & 0.163 \\
$\mathrm{a}$ & 0.0388 \\
\hline
\end{tabular}

Finally, The physical properties value from Table 2 were forecasted its HAT-P-12b model by the Star and Exoplanet View program, as shown in Figure 5. 


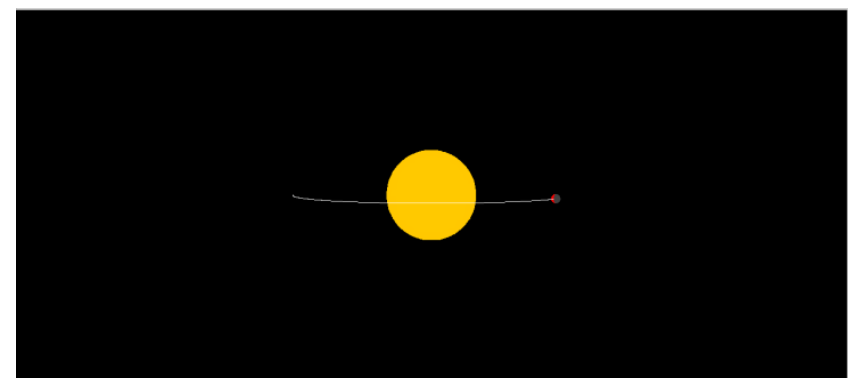

Fig .5. The exoplanet HAT-P-12b model.

\section{Conclusion}

This research analyzed 317 images of the exoplanet HAT-P-12b on April 14, 2017 from the Sierra Remote Observatories, the National Astronomical Research Institute of Thailand (Public Organization). The light curve was created and used to computed the physical properties of the planetary system HAT-P-12b by AstroImageJ software. The results show that the radius of the HAT-P-12b is around $0.951 \mathrm{R}_{\mathrm{J}}$. Its orbital period is 3.2105 days. The transit epoch Tc is 2457857.166110000 (BJD) and the transit duration $\mathrm{T}$ is 0.093519 days. Finally, theexoplanet HAT-P-12b model show that the decreasing brightness about $1.94864 \%$ and far from the HAT-P-12 center is $0.0388 \mathrm{AU}$.

\section{Acknowledgment}

The authors would like to thanks to Mr Torik Hengpiya, the National Astronomical Research Institute of Thailand (Public Organization) for this observation.

\section{References}

Planet Patrol (project). (2020), https://en.wikipedia.org/wiki/Planet_Patrol_(project).

David.F.Mota., and Marcelo.Vargas. (2017). Extrasolar planets as a probe of modified gravity. Physics Letters B, $769,485-490$

M.Godolt., J.I.Grenfell., A.Hamann-Reinus., D.Kitzmann., and M.Kunze. (2015). 3D climate modeling of Eartlike extrasolar planets orbiting different types of host stars. Planettary and Space Scince, 111, 62-76

Aomawa.L.Shields., SarahBallard., and JohnAsherJohnson. (2016). The habitability of planets orbiting M-dwarf stars. Physics Reports, 61, 1-38

Gour.Chandra.Paul., Sukumar.Senthilkumar., and Hafiiur.Rahman. (2020). On the implementation of novel RKARMS $(4,4)$ algorithm to study the structures of initial extrasolar giant protoplanets. Heliyon, 6, 25-65 